\title{
Elastoplastic and Damage Analysis of Trusses Subjected to Cyclic Loading
}

\author{
Dragoslav Šumarac ${ }^{1, a^{*}}$, Zoran Perović ${ }^{1, b}$ \\ ${ }^{1}$ Faculty of Civil Engineering, University of Belgrade, Serbia \\ asumi@eunet.rs, bzperovic@grf.rs
}

Keywords: cyclic plasticity, damage, trusses

\begin{abstract}
In the present paper, the Preisach model of hysteresis is extended to structural analysis of trusses damaged under cyclic loading in plastic range. Parameters for the Preisach model of cyclic plasticity are obtained from uniaxial loading experiment. Damage, as a consequence of micro cracks appearance due to alternating loading in plastic domain, is modeled using brittle elements according to Preisach procedure. Results of this research are compared with the already existing in the literature. In the paper examples of trusses under various cyclic loadings are presented.
\end{abstract}

\section{Introduction}

Since there are numerous examples of hysteresis phenomena occurring in physical processes (hysteresis in continuum mechanics, in ferromagnetism, in filtration through porous media etc.), appropriate modeling of hysteresis is of great interest for engineers and physicists. One of the most powerful scalar model of hysteresis, among those that are known so far, was proposed by the physicist F. Preisach in 1935 [1] to represent scalar ferromagnetism. Application of the Preisach model to cyclic behavior of elasto-plastic material was introduced in 1993 by [2,3]. One of the most important properties of the Preisach operator is the so-called memory map [4,14], but in addition it is shown in [2] that suggested (Preisach) model also possesses congruency and wiping out property, which makes this model $[2,3,4,6]$ appropriate to describe hysteretic behavior of elasto-plastic material. Although there are numerous well known models of cyclic plasticity defined [8,11], as is shown in [5], in this paper for uniaxial stress state, hysteresis will be defined, based on experimental data, in one particular rigorously mathematical form and implemented in finite element equations for trusses. In this paper, damage will be included in the analysis of trusses subjected to cyclic loading using two approaches. While in the first method, damage is incorporated in the threeelement unit [2,5] by adding damage element, in the second approach, damage is included in elastoplastic analysis by taking into account basic concepts of continuum damage mechanics. In this paper analysis is limited to a small deformations and quasi-static problems for uniaxial stress state.

Using finite element method, equilibrium equations for structural analysis of trusses are obtained and algorithm for numerical solution is defined in $\mathrm{C}++$ code. Several numerical examples will be analyzed and results obtained by suggested models will be presented. The second part of this paper contains basic outline of the Preisach model and its application of modeling ductile materials subjected to cyclic loading, as explained in $[2,3,4]$. In the third part, two different approaches, for including damage in the analysis of cyclic plasticity using the Preisach model, are presented. In the fourth part, finite element equations for static nonlinear analysis of trusses subjected to cyclic loading will be shown. In the fifth part, numerical examples are presented and results, obtained by this model, are analyzed and compared.

\section{Preisach model for cyclic plasticity}

According to Mayergoyz [9], the Preisach model implies the mapping of an input $u(t)$ on the output $f(t)$ in the integral form:

$$
f(t)=\iint P(\alpha, \beta) G_{\alpha, \beta} u(t) d \alpha d \beta
$$


where $G_{\alpha, \beta}$ is an elementary hysteresis operator given in Fig. 1a. Parameters $\alpha$ and $\beta$ are up and down switching values of the input, while $P(\alpha, \beta)$ is the Preisach function, i.e., a weight (Greens) function of the hysteresis nonlinearity to be represented by the Preisach model. The domain of integration of integral in the Eq. 1 is right triangle in the $\alpha, \beta$ plane, with $\alpha=\beta$ being the hypotenuse and $\left(\alpha_{0}, \beta_{0}=-\alpha_{0}\right)$ being the triangular vertex (Fig. 1b).

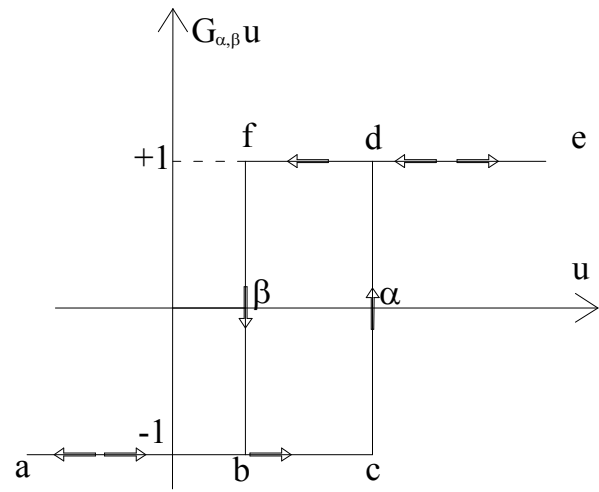

Fig.1 a Elementary hysteresis operator

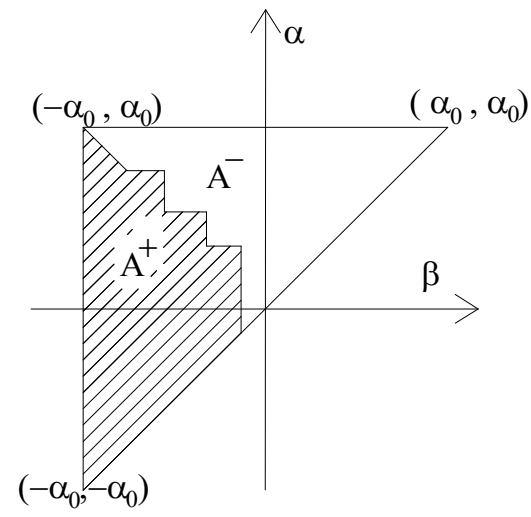

Fig. 1b Staircase line in the Preisach triangle

History of loading corresponds to staircase line $L(t)$, which divides triangle into two parts [2]. Elastic-linearly hardening material behavior, characterized by the stress-strain curve shown in Fig. 2a. ( $E$ and $E_{h}$ are elastic and hardening moduli, respectively), can be modeled by a three-element unit shown in Fig. $2 b$.

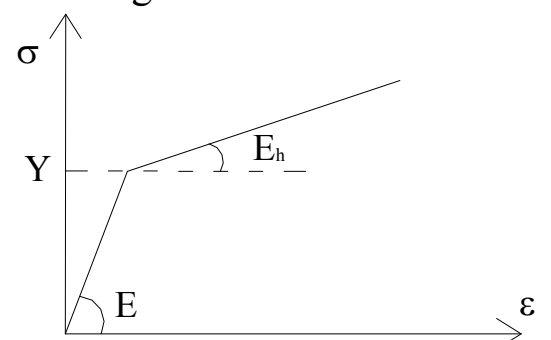

Fig. 2a Elastic-linearly hardening stress-strain behavior with elastic modulus $E$, initial yield stress $Y$ and hardening modulus $E_{h}$.

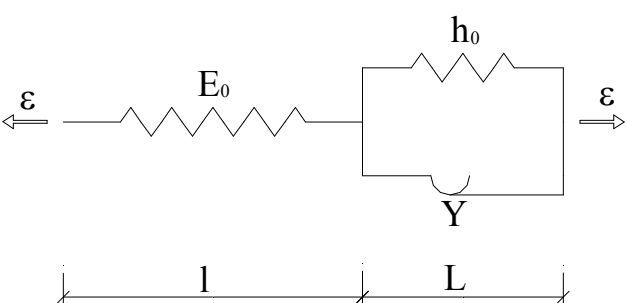

Fig. 2b Three-element unit reproducing the stress-strain behavior in (a)

One-dimensional hysteretic behavior of elastoplastic material can be successfully described by the Preisach model $[2,3,4]$. In the case when strain is used as an input function, for a system consisting of infinitely many of three-element units, connected in a parallel and with uniform yield strength distribution within the range $Y_{\min } \leq Y \leq Y_{\max }$, the total stress is

$$
\sigma(t)=\frac{E}{2}\left[\int_{-\varepsilon_{0}}^{\varepsilon_{0}} G_{\alpha, \alpha} \varepsilon(t) d \alpha-\frac{E-E_{h}}{2} \frac{1}{Y_{\max }-Y_{\min }} \iint_{A} G_{\alpha, \beta} \varepsilon(t) d \alpha d \beta\right]
$$

Comparison of results obtained via the Preisach and the Bouc-Wen model of hysteresis [14,15] were presented in [5].

\section{Modeling of damage}

In the present paper, beside the elastoplastic behavior, damage is also incorporated in the material response defined via the Preiach model of hysteresis. There will be two approaches of damage incorporation. In the first approach for including damage into cyclic plasticity of trusses, principle used in $[2,3]$, for the Preisach model of plastic hysteresis is used, resulting analytical solution in closed form for material behavior that includes both plasticity and damage in the uniaxial stress state. In the second approach, material behavior is defined by combination of a plasticity theory formulated using the Preisach model of hysteresis and an isotropic damage theory formulated in 
strain space. In this case, basic concepts of continuum damage mechanics are used, and solution is therefore obtained in the incremental-iterative procedure. In both approaches, microcracks closure effect in compression is taken in to account in such a way that the damage can increase only in tension. In this way this model is suitable for the quasi-brittle materials.

Preisach model for cyclic plasticity and damage. Damage can be included in three element unit $[2,3]$, using damage element with rupture limit $Y_{D}$, as shown in Fig.3b. Corresponding elastoplasic behavior is shown in Fig.3a. When tension stress in material reaches limit $Y_{D}$, complete failure of element is achieved.

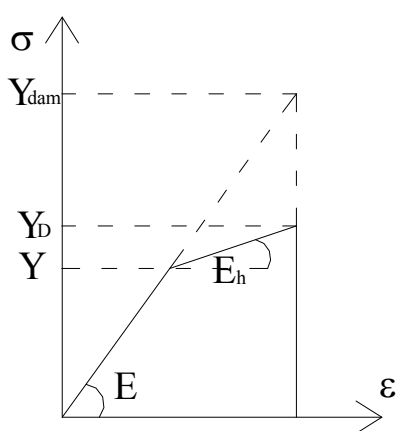

Fig. 3a Elastic-linearly hardening stress-strain behavior with elastic modulus $E$, initial yield stress $Y$ and hardening modulus $E$ h.

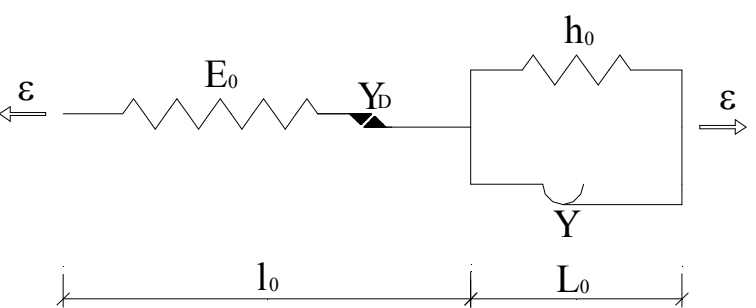

Fig. 3b Three-element unit reproducing the stress-strain behavior in Fig. 3a with stress limit for damage $Y_{\mathrm{D}}$.

Preisach function $P(\alpha, \beta)$ can be determined in this case in the same manner as presented in [2,3]:

$$
\begin{aligned}
& f_{\alpha, \beta}=f_{\alpha}-E(\alpha-\beta) \quad \text { for } \quad \alpha-2 \frac{Y}{E}-\beta<0 \\
& f_{\alpha, \beta}=f_{\alpha}-2 Y-E_{h}\left(\alpha-\beta-2 \frac{Y}{E}\right) \quad \text { for } \quad \alpha-2 \frac{Y}{E}-\beta \geq 0 \\
& f_{\alpha, \beta}=f_{\alpha}-Y_{D}=0 \quad \text { for } \quad \alpha-2 \frac{Y}{E}-\beta>0, \alpha \geq Y_{d a m} / \mathrm{E} . \\
& \frac{\partial f_{\alpha, \beta}}{\partial \beta}=E \cdot H(\alpha-\beta)-\left(E-E_{h}\right) \cdot H\left(\alpha-\beta-2 \frac{Y}{E}\right) \\
& -E_{h} \cdot H\left(\alpha-\beta-2 \frac{Y}{E}\right) \cdot H\left(\alpha-Y_{d a m} / E\right)+Y_{D} \cdot \delta\left(\alpha-Y_{d a m} / E\right) . \\
& P(\alpha, \beta)=\frac{1}{2} \frac{\partial^{2} f_{\alpha, \beta}}{\partial \beta \partial \alpha}=\left[E \cdot \delta(\alpha-\beta)-\left(E-E_{h}\right) \cdot \delta\left(\alpha-\beta-2 \frac{Y}{E}\right)-Y_{D} \cdot \delta\left(Y_{d a m} / E-\alpha\right)-\right. \\
& \left.E_{h} \cdot \delta\left(\alpha-\beta-2 \frac{Y}{E}\right) \cdot H\left(\alpha-Y_{\text {dam }} / E\right)-E_{h} \cdot H\left(\alpha-\beta-2 \frac{Y}{E}\right) \cdot \delta\left(\alpha-Y_{\text {dam }} / E\right)\right] / 2 .
\end{aligned}
$$

After determination of the Preisach function, output (stress) function can be expressed as a function of input (strain) function:

$$
\begin{aligned}
& \sigma(t)=\iint_{\alpha \geq \beta} P(\alpha, \beta) G_{\alpha, \beta} \varepsilon(t) d \alpha d \beta=\frac{E}{2} \int_{-u 0}^{u 0} \int_{-u 0}^{\alpha} \delta(\alpha-\beta) \cdot G_{\alpha, \beta} \varepsilon(t) d \alpha d \beta- \\
& \frac{E-E_{h}}{2} \int_{-u 0}^{u 0} \int_{-u 0}^{\alpha} \delta\left(\alpha-\beta-2 \frac{Y}{E}\right) \cdot G_{\alpha, \beta} \varepsilon(t) d \alpha d \beta- \\
& \frac{E_{h}}{2} \int_{-u 0}^{u 0} \int_{-u 0}^{\alpha} \delta\left(\alpha-\beta-2 \frac{Y}{E}\right) \cdot H\left(\alpha-Y_{d a m} / E\right) \cdot G_{\alpha, \beta} \varepsilon(t) d \alpha d \beta-
\end{aligned}
$$




$$
\begin{aligned}
& \frac{E_{h}}{2} \int_{-u 0}^{u 0} \int_{-u 0}^{\alpha} H\left(\alpha-\beta-2 \frac{Y}{E}\right) \cdot \delta\left(\alpha-Y_{d a m} / E\right) \cdot G_{\alpha, \beta} \varepsilon(t) d \alpha d \beta- \\
& \frac{1}{2} \int_{-u 0}^{u 0} \int_{-u 0}^{\alpha} Y_{D} \cdot \delta\left(\alpha-Y_{d a m} / E\right) \cdot G_{\alpha, \beta} \varepsilon(t) d \alpha d \beta .
\end{aligned}
$$

Dirac function under the corresponding limit of each integral determines where corresponding integral expression can exist. For a system consisting of infinitely many three-element units (Fig.2.b), connected in parallel, with uniform yield strength distribution $Y_{\min } \leq Y \leq Y_{\max }$, and with uniform damage strength distribution $Y_{D 1} \leq Y_{D} \leq Y_{D N}$, (Fig.4) total stress can be calculated as:

$$
\begin{aligned}
& \sigma(t)=\frac{E}{2} \int_{-u_{0}}^{u_{0}} G_{\alpha, \alpha} \varepsilon(t) d \alpha-\frac{E-E_{h}}{2\left(Y_{\max }-Y_{\min }\right)} \int_{Y_{\min }}^{Y_{\max }}\left[\int_{2 \frac{Y}{E}-u_{0}}^{\alpha} G_{\alpha, \alpha-2 \frac{Y}{E}} \varepsilon(t) d \beta\right] d Y- \\
& \frac{E_{h}}{2\left(Y_{D N}-Y_{D 1}\right)} \int_{Y_{D 1}}^{Y_{D N}}\left[\int_{2 \frac{Y}{E}-u_{0}}^{\alpha} G_{\alpha, \alpha-2 \frac{Y}{E}}^{\alpha} \varepsilon(t) d \beta \cdot H\left(\alpha-Y_{d a m} / E\right)\right] d Y_{D}- \\
& \frac{E_{h}}{2\left(Y_{D N}-Y_{D 1}\right)} \int_{Y_{D 1}}^{Y_{D N}}\left[\int_{-u_{0}}^{\alpha} G_{Y_{d a m} / E, \beta} \varepsilon(t) d \beta \cdot H\left(\alpha-\beta-2 \frac{Y}{E}\right)\right] d Y_{D}- \\
& \frac{1}{2\left(Y_{D N}-Y_{D 1}\right)} \int_{Y_{D 1}}^{Y_{D N}} Y_{D}\left[\int_{-u_{0}}^{\alpha} G_{Y_{d a m} / E, \beta} \varepsilon(t) d \beta \cdot\right] d Y_{D} \cdot
\end{aligned}
$$
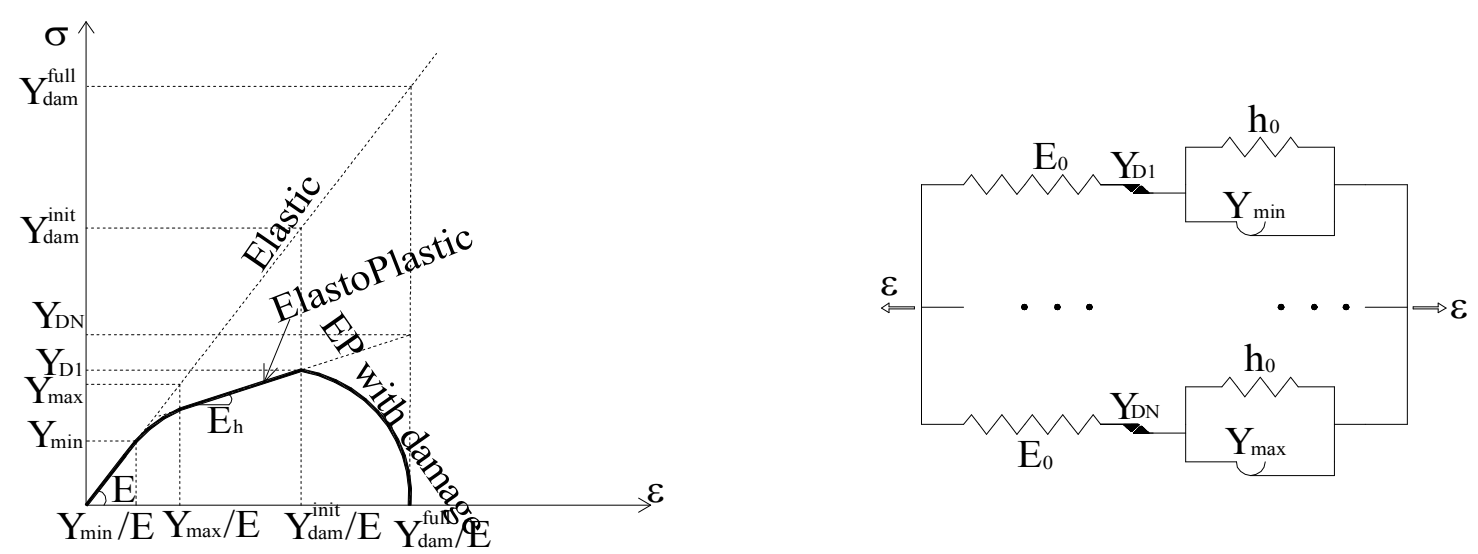

Fig. 4a Stress-strain behavior of material modeled Fig. 4b Parallel connection of infinite number by parallel connection of infinite number of of three-element units three-element units.

$$
\begin{aligned}
& \sigma(t)=\frac{E}{2} \int_{-u_{0}}^{u_{0}} G_{\alpha, \alpha} \varepsilon(t) d \alpha-\frac{E-E_{h}}{2\left(Y_{\max }-Y_{\min }\right)} \int_{Y_{\min } / E}^{Y_{\max } / E}\left[\int_{\frac{Y}{E}-u_{0}}^{\alpha} G_{\alpha, \alpha-2 \frac{Y}{E}} \varepsilon(t) d \beta\right] d \alpha \cdot \frac{E}{2}- \\
& \frac{E_{h}}{2\left(Y_{D N}-Y_{D 1}\right)} \int_{Y_{\text {dati }}^{\text {init }} / E}^{Y_{\text {dal }}^{\text {ful } / E}}\left[\int_{2 \frac{Y}{E}-u_{0}}^{\alpha} G_{\alpha, \alpha-2 \frac{Y}{E}} \varepsilon(t) d \beta \cdot H\left(\alpha-Y_{d a m} / E\right)\right] d \alpha \cdot E_{h}-
\end{aligned}
$$




$$
\begin{aligned}
& \frac{E_{h}}{2 \frac{E_{h}}{E}\left(Y_{d a m}^{\text {full }}-Y_{\text {dam }}^{\text {init }}\right)} \int_{Y_{\text {dain }}^{\text {init } / E}}^{Y_{\text {dall }}^{\text {ful }} / E}\left[\int_{-u_{0}}^{\alpha} G_{Y_{\text {dam }} / E, \beta} \varepsilon(t) d \beta \cdot H\left(\alpha-\beta-2 \frac{Y}{E}\right)\right] d \alpha \cdot E_{h}- \\
& \frac{1}{2 \frac{E_{h}}{E}\left(Y_{d a m}^{\text {full }}-Y_{d a m}^{\text {init }}\right)} \int_{Y_{d a m}^{\text {init }} / E}^{Y_{\text {dal }}^{\text {ful }} / E} Y_{D}\left[\int_{-u_{0}}^{\alpha} G_{Y_{\text {dam }} / E, \beta} \varepsilon(t) d \beta \cdot\right] d \alpha \cdot E_{h} .
\end{aligned}
$$

After further derivation, final expression for stress will be:

$$
\begin{aligned}
& \sigma(t)=\frac{E}{2} \int_{A 1} \int_{0} G_{\alpha, \beta} \varepsilon(t) d \alpha d \beta- \\
& \frac{E \cdot\left(E-E_{h}\right)}{4\left(Y_{\max }-Y_{\min }\right)} \int_{A 2} \int_{\alpha, \beta} \varepsilon(t) d \alpha d \beta-\frac{E_{h}^{2}}{2\left(Y_{D N}-Y_{D 1}\right)} \int_{A 3} \int_{\alpha, \beta} \varepsilon(t) d \alpha d \beta- \\
& \frac{E_{h}}{2\left(Y_{d a m}^{\text {full }} / E-Y_{d a m}^{\text {init }} / E\right)} \int_{A 4} \int G_{\alpha, \beta} \varepsilon(t) d \alpha d \beta-\frac{Y_{D 1}}{2\left(Y_{d a m}^{\text {full }} / E-Y_{d a m}^{\text {init }} / E\right)} \int_{A 5} \int G_{\alpha, \beta} \varepsilon(t) d \alpha d \beta .
\end{aligned}
$$

Where $A 2, A 3$ represent domains of integration and therefore, by geometrical interpretation, corresponding areas in the Preisach triangles (Fig. 5), while $A 1, A 4$ and $A 5$ denote only limits for integration:

$$
A 1: \alpha \geq \beta ; \quad A 4: \alpha \geq \beta, \alpha-\beta \geq 2 Y_{\text {dam }} / E, \alpha \geq Y_{\text {dam }} / E ; \quad A 5: \alpha \geq \beta, \alpha \geq Y_{\text {dam }} / E \text {. }
$$

Preisach triangle for domain of integration $A 2$ determines elastoplastic behavior as it is shown in $[2,3,4]$, and by geometrical interpretation, second part on the right hand side in (9) actually represents difference of positive and negative sets, $A^{+}$and $A^{-}$. Preisach triangle for domain of integration $A 3$ determines damage by degradation of moduli $E_{h}$ and $E$. Limit of damage initiation is defined by $Y_{d a m}^{\text {init }} / E(D=0)$, but this limit is increased by subsequent higher values of strain, that results in translation of lower limiting line in Fig.5.b. It can be said that $D$ is scalar damage variable $[15,16]$ that represents relation of number of ruptured elements $n$, and number of all elements $N$, $(D=n / N)$ in material defined by elements in Fig.4.b. Since this concept of damage refers only to tension stress, decreasing vertical line of the Preisach triangle does not have an effect on domain of integration $A 3$, therefore areas in corresponding Preisach triangle do not change in the case of compression.

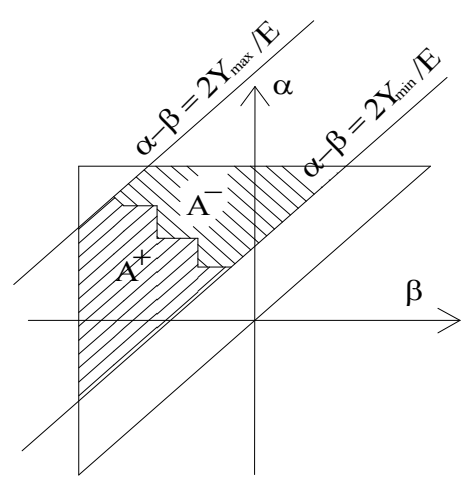

Fig. 5a Preisach triangle for domain of integration $A 2$

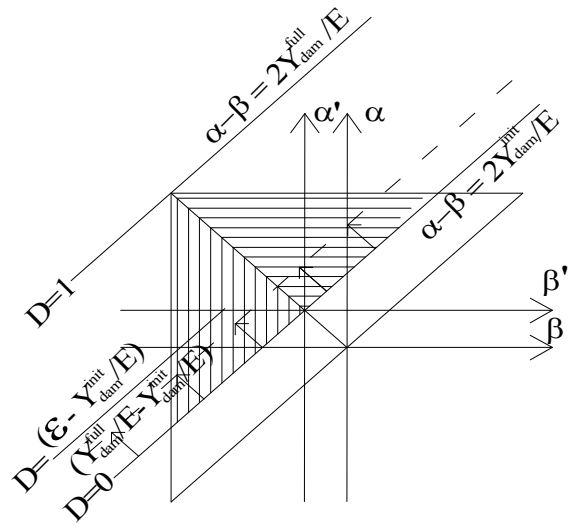

Fig. 5b Preisach triangle for domain of integration $A 3$

Although damage variable $D$ is constant through each step of loading in this approach, damage growth is achieved by accounting changes in the corresponding members of Eq.9.To illustrate this approach an example will be presented for uniaxial cyclic elastoplastic-damage behavior. For 
material properties and strain input history of load shown in Fig.6.a, resulting stress-strain curve is presented in Fig.6.b. In the first load step material is subjected to input strain level of $6 Y / E$, which, according to (9) results in output stress:

$$
\begin{aligned}
& \sigma(t)=\frac{E}{2}\left(\varepsilon_{1}-\left(-\varepsilon_{1}\right)\right)-\frac{E \cdot\left(E-E_{h}\right)}{4\left(Y_{\max }-Y_{\min }\right)}\left(\left(Y_{\max } / E-Y_{\min } / E\right)^{2} \cdot 2+\left(\varepsilon_{1}-Y_{\max } / E\right) \cdot\left(2 Y_{\max } / E-2 Y_{\min } / E\right)\right)- \\
& \frac{E_{h}{ }^{2}}{2\left(Y_{D N}-Y_{D 1}\right)}\left(\varepsilon_{1}-Y_{\text {dam }}^{\text {init }} / E\right)^{2} \cdot 2-\frac{E_{h}}{2\left(Y_{\text {dam }}^{\text {full }} / E-Y_{\text {dam }}^{\text {init }} / E\right)} \cdot 0-\frac{Y_{D 1}}{2\left(Y_{\text {dam }}^{\text {full }} / E-Y_{\text {dam }}^{\text {init }} / E\right)}\left(\varepsilon_{1}-Y_{\text {dam }}^{\text {init }} / E\right) \cdot 2= \\
& 0.975 Y .
\end{aligned}
$$

Level of strain at the end of the first step indicates $50 \%$ of damage in material $(D=0.5)$. In the second load step material is subjected to reversal strain to the level of $-4 Y / E$. Degradation of both elastic and hardening modulus is obtained in stress-strain curve on Fig. $6 \mathrm{~b}$. Stress at the end of this step is:

$$
\begin{aligned}
& \sigma(t)=\frac{E}{2}\left(\varepsilon_{2}-\left(-\varepsilon_{2}\right)\right)+D \cdot E\left(\varepsilon_{1}-\varepsilon_{2}\right) \\
& -\frac{E \cdot\left(E-E_{h}\right)}{4\left(Y_{\max }-Y_{\min }\right)}\left(\left(Y_{\max } / E-Y_{\min } / E\right)^{2} \cdot 2+\left(\varepsilon_{1}-Y_{\max } / E\right) \cdot\left(2 Y_{\max } / E-2 Y_{\min } / E\right)\right)- \\
& (1-D) \frac{E \cdot\left(E-E_{h}\right)}{4\left(Y_{\max }-Y_{\min }\right)}\left(\left(\varepsilon_{2}-\varepsilon_{1}\right) \cdot\left(2 Y_{\max } / E-2 Y_{\min }\right) \cdot 2+\left(2 Y_{\max } / E-2 Y_{\min }\right)^{2} \cdot 2\right)- \\
& \frac{E_{h}^{2}}{2\left(Y_{D N}-Y_{D 1}\right)}\left(\varepsilon_{1}-Y_{\text {dam }}^{\text {init }} / E\right)^{2} \cdot 2-\frac{E_{h}}{2\left(Y_{\text {dam }}^{\text {full }} / E-Y_{\text {dam }}^{\text {init }} / E\right)} \cdot 0 \\
& -\frac{Y_{D 1}}{2\left(Y_{\text {dam }}^{\text {full }} / E-Y_{\text {dam }}^{\text {init }} / E\right)}\left(\varepsilon_{1}-Y_{\text {dam }}^{\text {init }} / E\right) \cdot 2=-0.875 Y .
\end{aligned}
$$

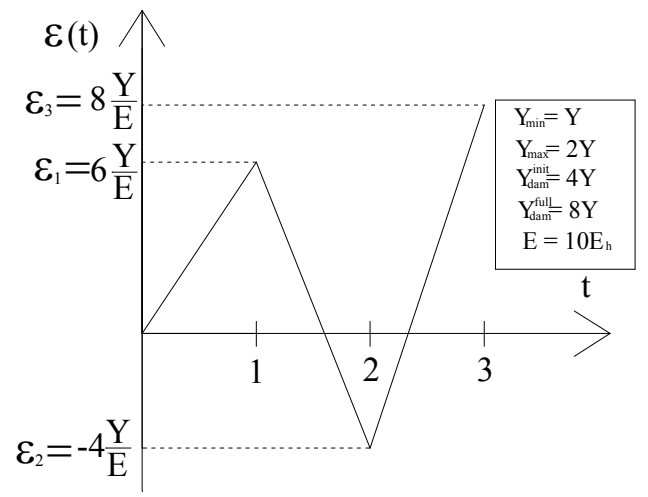

Fig. 6a Strain history for numerical example 1

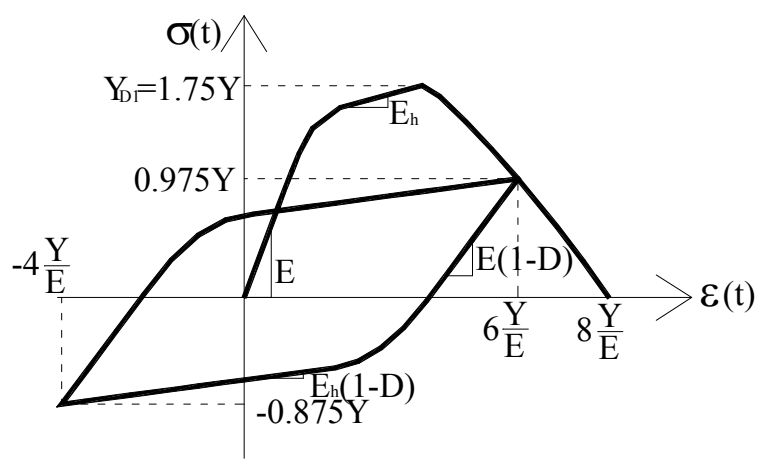

Fig. 6b Stress-strain curve for the first numerical example

In the third load step material is subjected to strain level of complete damage $8 Y / E$. Stress at the end of this step is:

$$
\begin{aligned}
& \sigma(t)=\frac{E}{2}\left(\varepsilon_{3}-\left(-\varepsilon_{3}\right)\right)+D \cdot E\left(\varepsilon_{1}-\varepsilon_{3}\right)- \\
& \frac{E \cdot\left(E-E_{h}\right)}{4\left(Y_{\max }-Y_{\min }\right)}\left(\left(Y_{\max } / E-Y_{\min } / E\right)^{2} \cdot 2+\left(\varepsilon_{1}-Y_{\max } / E\right) \cdot\left(2 Y_{\max } / E-2 Y_{\min } / E\right)\right)-
\end{aligned}
$$




$$
\begin{aligned}
& (1-D) \frac{E \cdot\left(E-E_{h}\right)}{4\left(Y_{\max }-Y_{\min }\right)}\left(\left(\varepsilon_{3}-\varepsilon_{1}\right) \cdot\left(2 Y_{\max } / E-2 Y_{\min }\right) \cdot 2\right)- \\
& \frac{E_{h}{ }^{2}}{2\left(Y_{D N}-Y_{D 1}\right)}\left(\varepsilon_{1}-Y_{d a m}^{\text {init }} / E\right)^{2} \cdot 2-\frac{E_{h}{ }^{2}}{2\left(Y_{D N}-Y_{D 1}\right)} \varepsilon_{3}\left(\varepsilon_{3}-\varepsilon_{1}\right) \cdot 2-\frac{E_{h} \cdot Y_{\text {dam }}^{\text {init }} / E \cdot\left(\varepsilon_{3}-\varepsilon_{1}\right)}{\left(Y_{\text {dam }}^{\text {full }} / E-\varepsilon_{1}\right)}- \\
& \frac{Y_{D 1}}{2\left(Y_{\text {dam }}^{\text {full }} / E-Y_{\text {dam }}^{\text {init }} / E\right)}\left(\varepsilon_{3}-Y_{\text {dam }}^{\text {init }} / E\right) \cdot 2=0 .
\end{aligned}
$$

Preisach model for cyclic plasticity coupled with CDM approach. In the following analysis basic concepts of macroscopic damage is introduced. Simple isotropic damage theory is implemented by introducing scalar damage measure in form of scalar variable $\omega$ that evolves from 0 (undamaged material) to 1 (fully damaged material):

$$
\sigma=(1-\omega) \hat{\sigma}
$$

where $\hat{\sigma}$ represents effective stress of undamaged body (in the case of elastic or elastoplastic analysis) and $\sigma$ represents actual stress caused by damage. Effective strain of undamaged body $\hat{\varepsilon}$ is considered to be equal to effective strain of damaged body $\varepsilon$. Since in the presented paper, uniaxial stress state is analyzed, with homogenous behavior of each element (bar) of structure (truss), local damage definition is sufficient and well suited for implementation since it is considered that damage is constant throughout each element of truss structure. Damage growth is only induced by tension stress. In this approach for including damage into analysis, the plasticity formulation remains based on the Preisach model of hysteresis [3]. Algorithm for elastoplastic analysis including damage can be defined as explained in [17] where this algorithm is used for gradient theories and for more complex stress state:

$$
\begin{aligned}
& d \hat{\sigma}=D^{e p} d \hat{\varepsilon} \\
& d \sigma=(1-\omega) D^{e p} \cdot d \varepsilon-d \omega \hat{\sigma} \\
& \sigma^{(i+1)}=\sigma^{(i)}+\left(1-\omega^{(i)}\right) D^{e p(i+1)} d \varepsilon-d \omega \hat{\sigma}^{(i)} \\
& D^{e p(i+1)} d \varepsilon=\hat{\sigma}^{(i+1)}-\hat{\sigma}^{(i)} .
\end{aligned}
$$

where $D^{e p}$ represents elastoplastic matrix of material in multiaxial stress state or tangent modulus in presented unixial case. Hence $D^{e p} d \varepsilon$ represents elastoplastic stress increment. Note that $\hat{\sigma}^{(i+1)}$ and $\hat{\sigma}^{(i)}$ represent effective stress of undamaged body in elastoplastic analysis at time increments $i+1$ and $i$ respectively, and they can be determined as presented in the second paragraph. Ductile damage variable $\omega$ can be defined as function of damage history parameter $\kappa^{d}$ and it grows from zero to one as the parameter $\kappa^{d}$ grows from threshold $\kappa_{o}$ to its ultimate value $\kappa_{u}$. Damage evolution can be defined as function that limits elastoplastic behavior in stress space and determines initiation of damage:

$$
f^{d}=\bar{\varepsilon}-\kappa^{d}
$$

where measure $\bar{\varepsilon}$ is the equivalent strain measure. It can be adopted as a function of elastic strains, or it could include plastic strains, which is more realistic and results in coupling of theories that include damage and plasticity (Fig.7.a.). It is important to emphasize that this algorithm Eq. 15-18 is incremental procedure, unlike procedure used in the first approach, where solution for damage in cyclic plasticity is defined as analytical solution in the closed form. The damage growth function 
governs damage variable evolution and it can be determined experimentally [18] in linear, power law or other form as shown in Fig.7b. In the following numerical analysis, modified power law form of damage variable evolution is used:

$$
\omega\left(\kappa^{d}\right)=1-\left(\frac{\kappa^{o}}{\kappa^{d}}\right)^{\beta}\left(\frac{\kappa^{u}-\kappa^{d}}{\kappa^{u}-\kappa^{o}}\right)^{\gamma} .
$$

where $\gamma, \beta$ represent material parameters. By varying these parameters, large number of failure modes induced by damage can be defined.

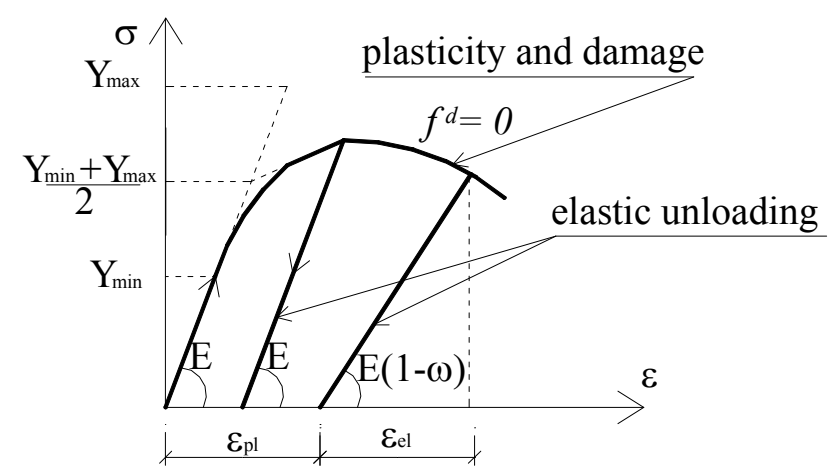

Fig. 7a Uniaxial elastoplastic behavior coupled with damage

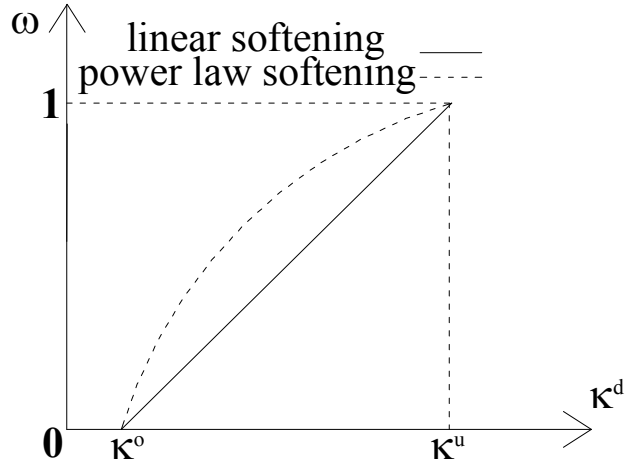

Fig. 7b Damage evolution law for damage variable $\omega$

\section{Finite element equations for trusses subjected to cyclic loading in plastic and damage domain}

Using principle of virtual displacements, equations for finite element procedures can be obtained. If only truss elements are considered, (body forces and surface forces are zero), only concentrated loads at nodes, as externally applied load are possible. In the finite element analysis we approximate the structure (in this case truss) as the assemblage of the discrete finite elements interconnected at the nodal points on the element boundaries. The expression for principle of virtual displacements then becomes:

$$
\sum_{m} \int_{V^{(m)}} \bar{\varepsilon}^{(m) T} \sigma^{(m)} d V^{(m)}=\sum_{i} \bar{u}^{i T} R_{C}^{i}
$$

where $\sigma$ represents stresses in equilibrium with applied loads, $R_{C}^{i}$ denotes concentrated forces on point $i$ of applied loads, $\bar{u}^{i}$ denotes virtual displacements, $\bar{\varepsilon}$ corresponding virtual strains and $m=$ $1,2 \ldots k$, where $k$ is the number of elements (bars). As it was presented in [5], if only one element $m$ of structure is analyzed, substituting equation (9) into (21), it is obtained:

$$
\begin{aligned}
& \int_{V} \bar{\varepsilon}^{T} \frac{E}{2}\left[\int_{A 1} G_{\alpha, \alpha} \varepsilon(t) d \alpha\right] d V-\int_{V} \bar{\varepsilon}^{T}\left[\frac{E \cdot\left(E-E_{h}\right)}{4\left(Y_{\max }-Y_{\min }\right)} \iint_{A 2} G_{\alpha, \beta} \varepsilon(t) d \alpha d \beta\right] d V \\
& \int_{V} \bar{\varepsilon}^{T}\left[\frac{E_{h}^{2}}{2\left(Y_{D N}-Y_{D 1}\right)} \iint_{A 3} G_{\alpha, \beta} \varepsilon(t) d \alpha d \beta\right] d V-\int_{V} \bar{\varepsilon}^{T}\left[\frac{E_{h}}{2\left(Y_{\text {dam }}^{\text {full }} / E-Y_{\text {dam }}^{\text {init }} / E\right)} \iint_{A 4} G_{\alpha, \beta} \varepsilon(t) d \alpha d \beta\right] d V \\
& \int_{V} \bar{\varepsilon}^{T}\left[\frac{Y_{D 1}}{2\left(Y_{\text {dam }}^{\text {full }} / E-Y_{\text {dam }}^{\text {init }} / E\right)} \iint_{A 5} G_{\alpha, \beta} \varepsilon(t) d \alpha d \beta\right] d V=\bar{u}^{T} R_{C} .
\end{aligned}
$$

If the second approach is used for defining damage, algorithm is presented in Eq. 15-18 and effective stress is calculated by accounting only first two elements of Eq.22. 
As it was shown in [5], second and third part in Eq.9 represent difference of integrals over positive and negative area $A^{+}(t)$ and $A^{-}(t)$ in corresponding Preisach triangle. It is obvious that area $A^{+}(t)$ is consisted of sum of $N$ trapezes whose vertices have coordinates equal to past input extrema [2], and therefore it represents function of predominant input strain data values $\left(\varepsilon_{t}^{x}, \varepsilon_{t}^{y}\right)$ (Fig.5.a):

$$
A^{+}(t)=\sum_{t=1}^{N}\left[\left(\varepsilon_{t}^{x}-\varepsilon_{t+1}^{x}\right)\left(\varepsilon_{t+1}^{y}-\varepsilon_{t+1}^{y^{*}}+\varepsilon_{t}^{y}-\varepsilon_{t}^{y^{*}}\right) / 2\right]
$$

Other parts on the left hand side of Eq.22 are linear functions of strain and they can easily be calculated in corresponding integration limits. Considering that displacement-based finite element method is used, it is necessary to exchange strain variable $\varepsilon$ with bar length change $\Delta u$, and because of Eq.23, $A^{+}(t)$ will therefore represent function of predominant input bar length change data values $\Delta u$. It is considered that this problem would not require large displacement and large strain analysis, and if strain displacement matrix $B$ is introduced, elastic nodal force vector and plastic (with damage) nodal force vector are defined [5]:

$$
\begin{aligned}
& \bar{u}^{(m) T}\left[\int_{V^{(m)}} B^{(m) T} E B^{(m)} d V^{(m)}\right] \bar{u}^{(m)}-\bar{u}^{(m) T}\left[\int_{V^{(m)}} B^{(m) T} \frac{E\left(E-E_{h}\right)}{4\left(Y_{\max }-Y_{\min }\right)} \frac{1}{\left(L^{(m)}\right)^{2}} d V^{(m)}\right] \cdot u_{A 2}^{(m)}- \\
& \bar{u}^{(m) T}\left[\int_{V^{(m)}} B^{(m) T} \frac{E_{h}^{2}}{2\left(Y_{D N}-Y_{D 1}\right)} \frac{1}{\left(L^{(m)}\right)^{2}} d V^{(m)}\right] \cdot u_{A 3}^{(m)}- \\
& \bar{u}^{(m) T}\left[\int_{V^{(m)}} B^{(m) T} \frac{E_{h}}{2\left(Y_{d a m}^{\text {full }} / E-Y_{d a m}^{\text {init }} / E\right)} \frac{1}{\left(L^{(m)}\right)} d V^{(m)}\right] \cdot u_{A 4}^{(m)}- \\
& \bar{u}^{(m) T}\left[\int_{V^{(m)}} B^{(m) T} \frac{1}{2\left(Y_{d a m}^{\text {full }} / E-Y_{d a m}^{\text {init }} / E\right)} \frac{1}{\left(L^{(m)}\right)} d V^{(m)}\right] \cdot u_{A 5}^{(m)}=\bar{u}^{(m) T} R_{C}^{i} \cdot \\
& R_{e l}^{(m)}-R_{p d}{ }^{(m)}=R_{C}^{i}
\end{aligned}
$$

Where elements $u_{A 2}^{(m)}$ to $u_{A 5}^{(m)}$ represent corresponding changes in domains of integration of each part. For the finite element assemblage, expression in Eq. 25 becomes:

$$
R_{e l}-R_{p d}=R
$$

It is important to emphasize that elements of vectors $R_{p d}$ represent both plastic and damage nodal forces transformed in the global system, by accounting elements on the left hand side of Eq.24. For solving problem of nonlinear static analysis, iterative procedure using Newton-Raphson initial stress method can be applied:

$$
\begin{aligned}
& K_{e l} \Delta U^{(i)}={ }^{t+\Delta t} R-{ }^{t+\Delta t} F^{(i-1)} \\
& { }^{t+\Delta t} U^{(i)}={ }^{t+\Delta t} U^{(i-1)}+\Delta U^{(i)} \\
& { }^{t+\Delta t} F^{(i)}=K_{e l}{ }^{t+\Delta t} U^{(i)}+{ }^{t+\Delta t} R_{d p}{ }^{(i)} .
\end{aligned}
$$

Procedure for iteration $i$ in Eq. 27 is repeated until convergence is achieved. According to defined procedures for numerical analysis from Eq. 27, algorithm for elastoplastic and damage analysis of trusses subjected to cyclic loading was defined in $\mathrm{C}++$ code. During every step and iteration in expressions given by Eq. 23 to Eq. 27, in every bar of truss structure, plastic and damage part from Eq. 26 is being calculated according to current state of corresponding bar and then assembled in global matrix in Eq. 26. In static analysis, if material has very small or no strain hardening $\left(E_{h} \approx 0\right)$, 
in order to provide some indication of when both the displacements and the forces are near their equilibrium values, it is recommended [7] that convergence criteria should be based on energy tolerance condition as shown in Eq. 28. In every iteration increment of internal energy is compared to initial internal energy increment:

$$
\Delta U^{(i) T}\left({ }^{t+\Delta t} R-{ }^{t+\Delta t} F^{(i-1)}\right) \leq \epsilon_{E}\left(\Delta U^{(1) T}\left({ }^{t+\Delta t} R-{ }^{t} F\right)\right)
$$

In the cases when load is displacement input history function, negative stiffness occurring in particular truss elements demands that convergence criteria should be based on both force and displacement tolerance condition. In the cases when load is force input history function, negative stiffness occurring in particular truss elements leads to divergence of numerical procedure.

\section{Numerical analysis}

For the numerical analysis, material property for structures is defined from the results of experiment for cyclic loading in stable cycle loop [10], which is used to determine analytical solution based on the Preisach model as shown in [5]. Different models used in the numerical analysis are shown in Fig.8, where parameters for damage models are adopted arbitrarily. Beside elastoplastic behavior defined using the Preisach model of hysteresis without damage, two models for incorporating damage in elastoplastic analysis are used. In the first approach, the Preisach model of hysteresis is also used to define damage in material as presented above in the first and the second approach. Paremeters $\kappa_{o}, \kappa_{u}, \alpha, \gamma$ are determined to match model of damage defined using the Preisach model of hysteresis as shown in Fig.8.b. It is adopted that the equivalent strain measure is based on total strain measure.

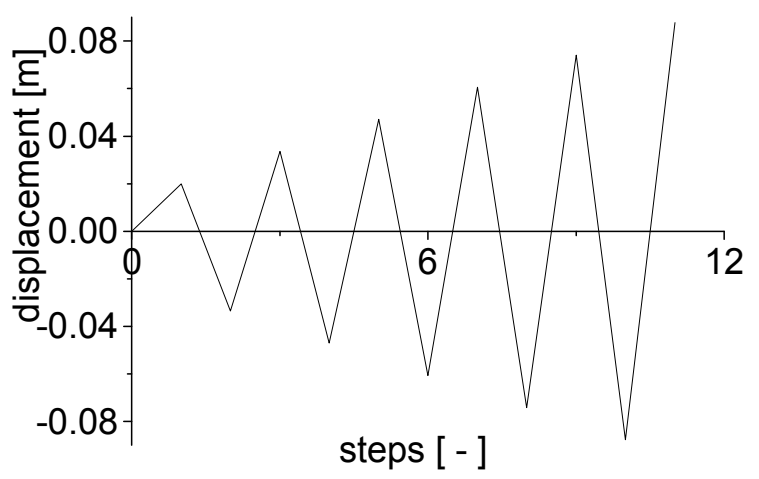

Fig. 8a Time history function of displacement

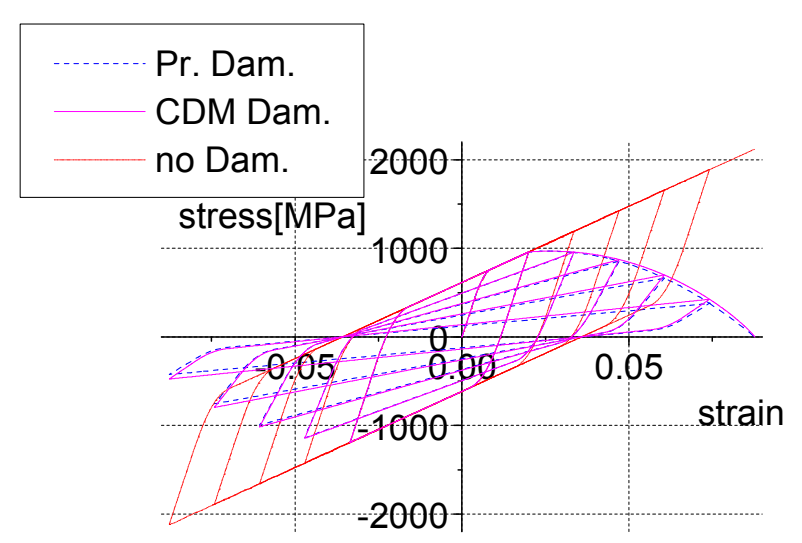

Fig. 8b Resulting hysteretic loops for different models

In order to emphasize the possibilities of presented models, in the first example, one bar (area $0.02 \mathrm{~m}^{2}$ ) was subjected to cyclic loading in plastic domain, where input functions for loading were displacement (Fig.8.a) and force history functions. When displacement history function is used for loading to the maximum damage limit, complete degradation of both elastic and hardening modulus is occurred. In the case where the Preisach model of hysteresis is used for defining damage, resulting bounding stress-strain curve can be obtained with single increment in each step, while algorithm defined in the second approach (CDM coupled with plasticity) demands high number of increments to achieve convergence of solution. When the force history function is used for loading, analysis is possible only until the strain limit of negative stiffness. Summary of the analysis conducted in the first numerical example is shown in the Table 1.

In the second numerical example, truss structure shown in the Fig.10. is subjected cyclic loading, where time history functions were displacement functions. Material property is identical as in the first numerical example. Each bar of truss structure has cross sectional area equal to $0.02 \mathrm{~m}^{2}$. 
Table 1. Overview of the Analysis in the first numerical example

\begin{tabular}{|c|c|c|c|c|c|c|}
\hline \multirow{2}{*}{ Analysis } & \multicolumn{3}{|c|}{ No.Ex.1 - Load $\mathrm{u}(\mathrm{t})$} & \multicolumn{3}{c|}{ No.Ex.1 - Load R(t) } \\
\cline { 2 - 8 } & $\begin{array}{c}\text { num. of } \\
\text { iterations }\end{array}$ & $\begin{array}{c}\text { num. of } \\
\text { increments }\end{array}$ & $\begin{array}{c}\text { CPU } \\
\text { time [s] }\end{array}$ & $\begin{array}{c}\text { num. of } \\
\text { iterations }\end{array}$ & $\begin{array}{c}\text { num. of } \\
\text { increments }\end{array}$ & $\begin{array}{c}\text { max displ. } \\
{[\mathrm{m}]}\end{array}$ \\
\hline EP An. w/o Dam. & 1 & 11 & 0.4 & 1074 & 11 & 0.020498 \\
\hline EP An. w/ Pr.Dam. & 1 & 11 & 0.5 & 3932 & 11 & 0.024588 \\
\hline EP An. w/ CDM.Dam. & 1 & 1579 & 7.47 & 10738 & 33 & 0.024458 \\
\hline
\end{tabular}

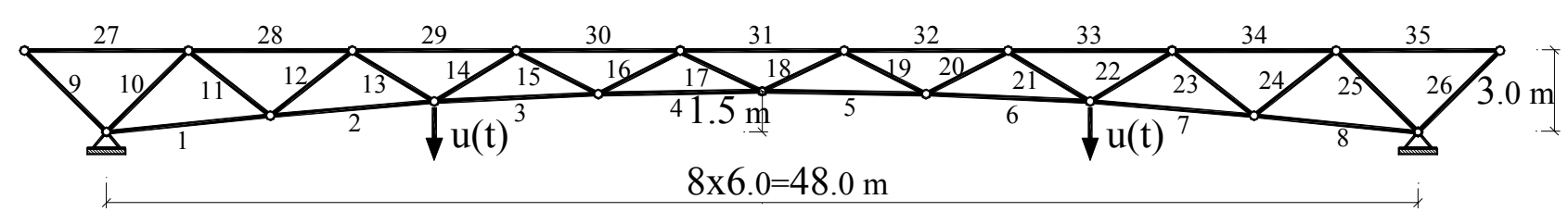

Fig. 10 Truss structure in the second numerical example

Time history function for displacement in the second example is shown in Fig. 11.a and resulting hysteretic loops for some of the characteristic bars are presented in the Fig. 11b. Model of damage defined using the Preisach model of hysteresis demands in this case iterations to perform redistribution of forces in truss structure, while model defined with coupling CDM approach with plasticity demands higher number of increments and consequently demands higher number of iterations to achieve convergence of solution. Summary of the analysis conducted in the second numerical example is shown in the Table 2.

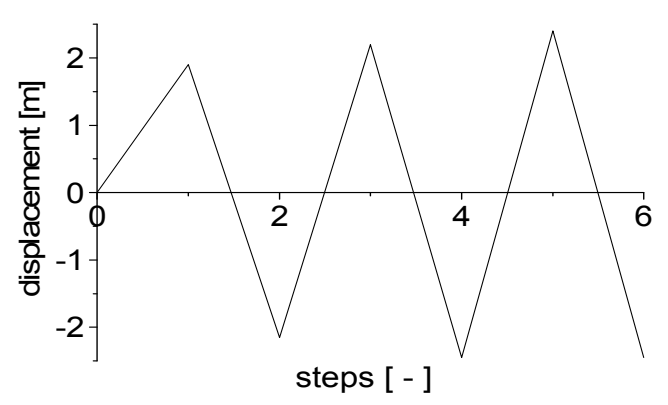

Fig. 11a Time history function of displacement in the second numerical example

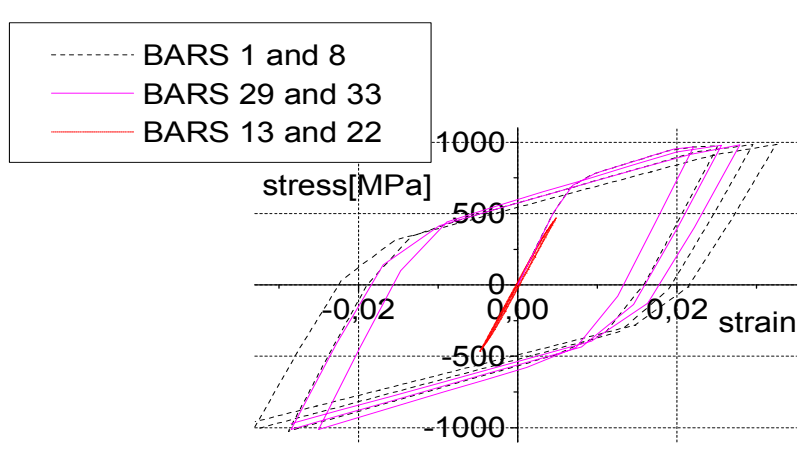

Fig. 11b Resulting hysteretic loops obtained via Preisach model for damage

Table 2. Overview of the Analysis in the second numerical example

\begin{tabular}{|c|c|c|c|c|c|}
\hline \multirow{2}{*}{ Analysis } & \multicolumn{5}{|c|}{ No.Ex.2 - Load u(t) } \\
\cline { 2 - 6 } & $\begin{array}{c}\text { num. of } \\
\text { iterations }\end{array}$ & $\begin{array}{c}\text { num. of } \\
\text { increments }\end{array}$ & $\begin{array}{c}\text { CPU time } \\
{[\mathrm{s}]}\end{array}$ & $\begin{array}{c}\text { max damage [\%] } \\
\text { bars 1 and 8 }\end{array}$ & $\begin{array}{c}\text { max damage [\%] } \\
\text { bars 29 and 33 }\end{array}$ \\
\hline EP An.w/o Dam. & 576 & 6 & 5.716 & - & - \\
\hline EP An. w/ Pr.Dam. & 2263 & 9 & 21.6 & 18.52 & 11.58 \\
\hline EP An. w/ CDM.Dam. & 64169 & 594 & 55.1 & 17.5 & 11.16 \\
\hline
\end{tabular}

\section{Conclusion}

In the present paper, the elastoplastic analysis of trusses subjected to cyclic loading is extended by taking damage into account. In the first approach, the Preisach model of hysteresis was used for defining both plasticity and damage in material, while in the second damage was defined by using basic concepts of continuum damage mechanics in the isotropic material. Although, the second approach enables various different failure modes obtained using different exponential parameters, for numerical analysis of truss structures, the first approach uses solution for material behavior, defined in closed form, leading to higher computational efficiency. For numerical analysis, critical 
points are those in which negative stiffness occurs, leading to redistribution of forces in opposite direction. In general case, energy, force and displacement criteria for convergence must be satisfied in order for solution to converge.

\section{References}

[1] F. Preisach, Über die magnetische Nachwirkung, Z . Phys., 94 (1935) pp. 277- 302.

[2] V.A. Lubarda, D. Sumarac and D. Krajcinovic, Hysteretic response of ductile materials subjected to cyclic loads, in: J.W. Ju (Ed.), Recent Advances in Damage Mechanics and Plasticity, ASME Publication, AMD, 123 (1992) pp. 145-157.

[3] V.A. Lubarda, D. Sumarac and D. Krajcinovic, Preisach model and hysteretic behavior of ductile materials. Eur. J. Mech., A/Solids, 12, (4) (1993) 445-470.

[4] D. Sumarac, S. Stosic, The Preisach model for the cyclic bending of elasto-plastic beams, Eur. J. Mech., A/Solids 15 (1) (1996) 155-172.

[5] D. Šumarac and Z. Perović, Cyclic plasticity of trusses, Archive of Applied Mechanics: DOI 10.1007/s00419-014-0954-7, Published online 03 dec 2014.

[6] D. Šumarac and Z. Petrašković, Hysteretic behavior of rectangular tube (box) sections based on Preisach model, Archive of Applied Mechanics, 82 (10) (2012) 1663-1673.

[7] K.J. Bathe, Finite Element Procedures, Prentice Hall, 1996.

[8] D.W. Iwan, On a class of models for the yielding behavior of continuous and composite systems, J. Appl. Mech., 34 (1967) 612-617.

[9] D.I. Mayergoyz, Mathematical Models of Hysteresis, Springer-Verlag, New York, 1991.

[10] D. Kujawski and E. Krempl, The Rate (Time)-Dependent Behaviour of Ti-7Al-2Cb-1Ta Titanium Alloy at Room Temperature Under Quasi Static Monotonic and Cyclic Loading, J. Appl. Mech., 48 (1981) 55-63.

[11] J.R. Asaro, Elastic-plastic memory and kinematic type hardening, Acta Metall. 23 (1975) $1255-1265$.

[12] R. Bouc, Modèle Mathématique d'Hystérésis, Acustica, 24 (1971) pp. 16-25 (inFrench).

[13] K.Y. Wen, Method for Random Vibration of Hysteretic Systems, Journal of the Engineering Mechanics Division, Proc. ASCE, 102 (1976) pp. 249-263.

[14] A. Visintin, Mathematical Models of Hysteresis, Dipartimento di Matematica dell'Universita degli Studi di Trento, Italia, 2005.

[15] Sumarac, D. and Krajcinovic, D.: "A Self-consistent Model for Microcrack-weakened Solids", Mechanics of Materials, 6, pp. 39-52, 1987.

[16] Sumarac,D. and Krajcinovic, D.: "A Mesomechanical Model for Brittle Deformation Processes", Part II, Journal of Applied Mechanics, 56, pp. 57-62, 1989.

[17] de Borst, R., Pamin, J., Geers, M.G.D.: On coupled gradient - dependent plasticity and damage theories with view to localization analysis, Eur. J.Mech. A/Solids, 18, 939-962, (1999)

[18] Panis, M.W., van der Slius, O., Peerlings, R.H.J.: Experimental identification of damage evolution law for steel, Eindhoven, (2004). 